\title{
Rassismus in klinischer Forschung
}

\section{Lasst uns den „Kaukasier" endlich begraben}

\author{
Die Pandemie offenbart einen latenten Rassismus europäischer \\ und nordamerikanischer Prägung. Das geht bis heute mit Defiziten \\ in der klinischen Forschung einher: Ethnizität ist als Faktor in \\ klinischen Studien unterrepräsentiert - und schwer zu fassen.
}

n seiner Kurzgeschichte „Die Virusepidemie in Südafrika“ beschreibt der Schweizer Schriftsteller Friedrich Dürrenmatt, wie der weiße Regierungspräsident des Apartheid-Regimes mit Schnupfen und Fieber erwacht. Kurze Zeit später muss er feststellen, dass seine Haut infolge der Virusinfektion dunkel geworden ist. Er wird verhaftet und ins Gefängnis geworfen. Dort befindet sich bereits sein Finanzminister, der infektionsbedingt ebenfalls zum "schwarzen Weißen" geworden ist. Chaos und Bürgerkrieg brechen aus, da niemand mehr weiß, wer ein „schwarzer Schwarzer“ und wer ein „schwarzer Weißer" ist ... die Apartheid soll aber unbedingt aufrechterhalten werden. Schließlich werden immer öfter Ehen zwischen „schwarzen Weißen“ und „schwarzen Schwarzen“ geschlossen und siehe da: etwa die Hälfte ihrer Kinder sind wieder weiß. Dabei gilt inzwischen die vom „schwarzen weißen“ Regierungspräsidenten ausgegebene
Parole „Schwarz ist unsere Hautfarbe, zu der wir uns bekennen." Allerdings seien nicht Weiße, die schwarze Weiße seien „wie wir“, sondern „weiße Schwarze“ eine neue Gefahr für die „südafrikanische Rasse“...

Dürrenmatt forciert auf satirische Weise das Durcheinander von weißen/ schwarzen Schwarzen, von schwarzen/ weißen Weißen derart, dass dem Leser schwindlig wird, während er sich unwillkürlich auflachend dem überraschenden Ende der Geschichte nähert. Ein Lachen, das einem im Halse stecken bleibt. Nicht nur, weil die Story die Absurdität rassistischen Denkens vor Augen führt, sondern weil die Verknüpfung der Themen Pandemie und Rassismus in der vor drei Jahrzehnten verfassten Geschichte geradezu prophetisch auf Ereignisse des Jahres 2020 verweist.

\section{Latenter Rassismus in der Medizin} Die COVID-19-Pandemie hat einmal mehr den Schleier über latent vorhan- denem Rassismus gelüftet, sei es in den USA, sei es in Südamerika oder sei es angesichts der Art und Weise, wie die reichen Industrieländer die COVID19-Impfstoffe global verteilen.

Rassismus, der nicht Halt macht vor dem medizinischen Betrieb und der latent auch die klinische Forschung betrifft. Diese findet nach wie vor bevorzugt in hellhäutigen Bevölkerungsgruppen statt, ungeachtet der multiethnischen Bevölkerungszusammensetzung in allen Industrieländern. Das Dilemma einer unzureichenden Erfassung ethnischer Kategorien wird offenbar.

Das Konzept der Existenz von „Menschenrassen" haben Wissenschaftler längst ad acta gelegt. Es ist nötig, das an dieser Stelle so klar zu schreiben, denn der Satz dürfte bis heute noch manchen überraschen. Aus medizinischer Sicht wäre es ja wunderbar, wenn phänotypische Eigenschaften wie Haut- und Augenfarbe, Schnitt des Gesichts oder die Haarstruktur auf objektive biologische oder pathogenetische Unterschiede schließen ließen. Dem ist aber nicht so. Tierzüchter sind zwar in der Lage, bestimmte Populationen von Hunden, Pferden oder Rindern zu kreieren. Das aber ist nicht das Ergebnis
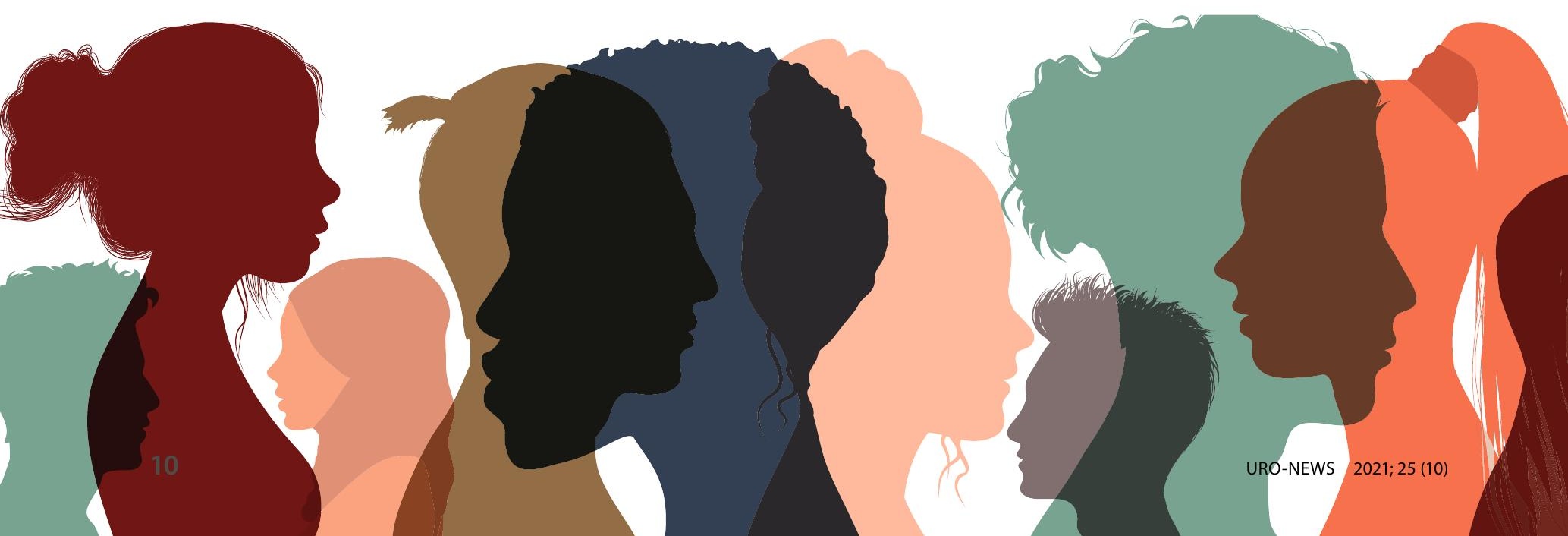
eines natürlichen, biologischen Prozesses.

„Denkschemata des biologisch begründeten Rassismus wie die Analogie zu Haustierrassen haben dazu verführt, anzunehmen, mit gleichem Recht von Menschenrassen („human races“) sprechen zu können“, heißt es in der „Jenaer Erklärung “, die das Max-Planck-Institut für Menschheitsgeschichte Ende 2019 veröffentlicht hat. Man habe angenommen, die Ähnlichkeit innerhalb einer vermeintlichen Menschenrasse sei wesentlich höher als zwischen diesen "Rassen“ und deshalb sei eine Abgrenzung möglich. „Ein bitterer Trugschluss", so die Wissenschaftler: Beim Menschen seien die größten genetischen Unterschiede innerhalb einer Population zu finden, nicht zwischen Populationen.

\section{Ethnisch definierte Subgruppen}

Hat sich das auch in der klinischen Wissenschaft herumgesprochen? Wird nicht in manchen Studien mehr oder weniger bewusst eine biologische Ursache von in ethnisch definierten Subgruppen gefundenen Unterschieden insinuiert?

Den Begriff „Rasse“ wird man in deutschsprachigen Publikationen zwar nicht mehr finden, in englischsprachigen Publikationen ist aber nach wie vor von „race and ethnicity“ die Rede. Eine Differenzierung, die sehr wohl darauf hinweist, dass versucht wird, äußerliche Typologien in Übereinstimmung mit womöglich vorhandenen „inneren“ Typologien zu bringen. Die Frage ist, inwiefern eine wie auch immer pigmentierte Haut deckungsgleich ist mit einer bestimmten Ethnie. Ein Begriff, der

\section{Definition von Rasse und Ethnizität}

Während in Deutschland ethnische Minderheiten vor allem mit dem Begriff „Migrant" beschrieben werden, dominieren in Ländern mit postkolonialer Migrationsgeschichte die Begriffe "Race“ (Rasse) und „Ethnicity" (Ethnizität).

"Rasse" war ursprünglich als ausschließlich biologisch bestimmte Kategorie angelegt, die sich an äußeren Merkmalen wie Hautfarbe, Körperbau und Haarstruktur orientierte, aber auch menschliche Verhaltensweisen als biologisch bedingt darstellte. Heute wird in englischsprachigen Ländern der Begriff "Race" um kulturelle und soziale Merkmale erweitert. „Race“ ist Teil der individuellen Identität, die sich unter anderem aus physischen Ähnlichkeiten und der Herkunft ergibt. Dementsprechend ordnen sich Bürger der USA oder des Vereinigten Königreiches selbst unter "White“, „Black“, „Asian“ oder "Hispanic" ein. Die Indikatoren dafür sind nicht einheitlich. Vor allem aber wird damit eine biologische und kulturelle Homogenität unterstellt.

"Race" und „Ethnicity" werden zudem häufig synonym verwendet, unter Umständen in der Formulierung "race-ethnicity". Das deutet darauf hin, dass die Nutzer der Termini selbst keine trennscharfe Linie zwischen diesen Begriffen ziehen.

Nach Max Weber (1864-1920) beschreibt der Begriff „Ethnizität" eine Gruppe von Menschen gleicher Abstammung und Kultur. Es gibt Gemeinsamkeiten bezüglich Sprache, Lebensführung, Traditionen, Werte, Religion. Dies erzeugt ein subjektives Zugehörigkeitsgefühl, unabhängig von einer biologischen Abstammung. Es handelt sich damit um eine soziale Kategorie. Wird in Publikationen der Begriff "ethnicity“ gebraucht, kann dieser jedoch auch biologische Differenzen implizieren. Dies erschwert die Operationalisierbarkeit des Begriffs „Ethnie“ in Studien [Bundesgesundheitsbl. 2006;49:85360].

nicht biologisch definiert ist, sondern, knapp formuliert, eine kulturelle Identität beschreibt.

Nehmen wir den nach wie vor in medizinischen Publikationen gängigen Begriff „Kaukasier“ (engl.: „caucasian“). Er beschreibt, wissenschaftlich verbrämt, nichts anderes als Menschen mit heller Haut. Der Terminus wurde vor über 200 Jahren vom deutschen Anthropologen Johann Friedrich Blumenbach (1752-1840) geprägt. Blumenbach bezeichnete damit europäische Populationen und grenzte davon Asiaten, Afrikaner, Amerikaner sowie „Malayische“ (Südostasien, Polynesien, Australien) ab.
Interessanterweise stellte Blumenbach bereits 1775 in seiner Dissertation fest, dass diese Unterschiede rein äuBerlicher Natur seien. Klare Grenzen zwischen den Populationen zu ziehen, sei nicht möglich. Bekanntlich sahen das Rassentheoretiker Ende des 19. und Anfang des 20. Jahrhunderts ganz anders. Das hat Folgen - bis heute.

Es ist üblich, dass sich Teilnehmer an klinischen oder soziologischen Studien, etwa in den USA oder in Großbritannien, auf Fragebögen selbst als „Black“, „Asian“, „White“ oder „Mixed“ klassifizieren. Manchmal wird noch differenziert, ob man selbst oder die Vorfahren 
aus Indien, Bangladesch oder China stammen, ob Weiße britischer, irischer oder westeuropäischer Herkunft sind, Schwarze aus Afrika oder aus der Karibik stammen.

Wählen wir die Kategorie "Schwarz" (Black). Frage: Wo würde US-Vizepräsidentin Kamala Harris wohl ihr Kreuz machen, wenn sie, sagen wir, an einer Hypertonie-Studie teilnehmen würde? Ihre Mutter stammt aus dem indischen Madras, ihr Vater aus Jamaika. Sie gilt als erste Afroamerikanerin auf diesem Posten. Ist sie Afroamerikanerin? Ist sie
Afroasiatin? Und würde das einen Unterschied machen?

Die Vorfahren der Afroamerikaner stammen überwiegend aus südlich der Sahara gelegenen Gebieten Afrikas. Zwar stammen $90 \%$ der Jamaikaner von afrikanischen Sklaven des 17. und 18. Jahrhunderts ab, „den Afrikaner“ gibt es aber gar nicht. Geradezu paradox sei diese Bezeichnung, erklärt die Max-Planck-Gesellschaft für Menschheitsgeschichte in ihrer erwähnten Erklärung. Menschen aus Ostafrika seien näher verwandt mit Menschen von au-

\section{„Rasse" - die traurige Chronik eines wissenschaftlichen Irrwegs}

"Rasse" als Begriff für Menschengruppen ist erstmals schriftlich für die spanische Reconquista Ende des 15. Jahrhunderts belegt. Er wurde zur Bezeichnung von Menschen jüdischer oder maurischer „Abstammung" benutzt [Brückmann T et al. Rassifizierte Gene: Zur Aktualität Biologischer "Rasse"]. Die Naturwissenschaften griffen die Vorstellung natürlicher Entwicklungsstufen der "Völker" auf, angefangen von sogenannten "Wilden" hin zur Ausbildung "höherer" Zivilisationen. Die erste wissenschaftliche Verwendung des Klassifikationsbegriffs "Rasse“ wird dem französischen Arzt François Bernier (1620-1688) zugeschrieben. In seinem Text "Nouvelle division de la Terre, par les differentes Espèces ou Races d'hommes qui I'habitent" berichtet er von einer zwölfährigen Reise und typologisiert die von ihm beobachteten Menschen. Johann Friedrich Blumenbach (1751-1840) teilte die Menschen in fünf Rassen ein. Der von ihm geprägte Begriff "Kaukasier“ wird bis heute verwendet. Carl von Linné (1707-1778) unterschied anhand von Hautfarbe, Haaren, Charakter, Temperament, Geist und Kleidung vier Varietäten. Ebenso sprach und schrieb Immanuel Kant (1724-1804) "von den verschiedenen Racen der Menschen". In "ihrer größten Vollkommenheit" sei die "Race der Weißen" zu sehen. Charles Darwin (1809-1882) griff Kants Vorstellungen auf und äußerte die Überzeugung, dass in „einer künftigen Zeit [...] die zivilisierten Rassen der Menschheit wohl sicher die wilden Rassen auf der ganzen Erde ausgerottet und ersetzt haben" würden.

Auf diesen Grundlagen entwickelte sich im 19. Jahrhundert die "Rassen-Anthropologie" und damit eine "wissenschaftliche" Basis für rassistische Ausbeutungs- und Exklusionspraktiken. Verbreitet war die Überzeugung, die Erbmasse ganzer Bevölkerungsgruppen könne sich durch erbliche Tendenzen verbessern, aber auch verschlechtern [Blom P. Der taumelnde Kontinent]. So meinte der britische Universalgelehrte Francis Galton (1822-1911), die gesellschaftlichen Erfolge wichtiger Familien, die hohe Beamte, Politiker, Wissenschaftler und Künstler hervorgebracht hatten, ließe sich nur mit deren inhärenten Eigenschaften erklären. Dieses Erbmaterial müsse reingehalten werden. Galton war es, der aus dem griechischen Wort für "wohlgeboren" den Begriff „eu-genisch" formte. In seinem Werk „Hereditary Genius" beschrieb er eine Rasse von Übermenschen. Eugenik bedeutete nichts anderes, als durch wohlausgewählte Ehen besonders wertvolle Menschen zu zeugen, die die Probleme dieser Welt lösen würden.

In Deutschland war es vor allem Ernst Haeckel (1834-1919), dessen

populärwissenschaftliche Werke zu Bestsellern wurden. Der Begründer der Stammesgeschichtsforschung habe "durch seine vermeintlich wissenschaftliche Anordnung von ,Menschenrassen' in einem ,Stammbaum' in fataler Weise zu einem angeblich wissenschaftlich begründeten Rassismus beigetragen", so die Max-PlanckGesellschaft für Menschheitsgeschichte in ihrer "Jenaer Erklärung" vom September 2019. Diese Verwissenschaftlichung ist als entscheidende Voraussetzung für den folgenden Rassenwahn des deutschen Nationalsozialismus zu sehen. Auch nach 1945 wurden eugenische Thesen noch lange Zeit in Parlamenten vertreten, an Universitäten gelehrt, in Schriften verbreitet, die hohe Auflagen erreichten - und sie sind bis heute in etlichen Köpfen präsent.

Dr. Thomas Meißner/Markus Seidl

ßerhalb Afrikas als mit Menschen aus Südafrika. Das Gedankenspiel der Teilnahme an einer klinischen Studie ließe sich beliebig fortsetzen, etwa mit einem Amerikaner japanischer Herkunft, dessen Großvater nach dem Zweiten Weltkrieg eingewandert ist.

Auch für Deutschland ist das inzwischen relevant, weil es längst zum Einwanderungsland geworden ist: Etwa ein Viertel der Bürgerinnen und Bürger haben einen Migrationshintergrund. Die Wahrscheinlichkeit ist hoch, dass Millionen Menschen auf diesem Planeten auf einem Fragebogen mit mehr oder weniger willkürlich gewählten typologischen Konstrukten unsicher wären, wo das Kreuz richtig gesetzt wäre. Dennoch nehmen Leser wissenschaftlicher Originalien statistische Analysen ethnischer Subgruppen als objektive Wahrheiten hin. Wie die ethnische Gruppierung zustande gekommen ist, dazu finden sich oft keine Angaben.

Selbstverständlich gibt es mehr oder weniger gesicherte Unterschiede zwischen Bevölkerungsgruppen im Auftreten und der Morbidität bestimmter Krankheiten oder beim Ansprechen auf bestimmte Medikamente. Nur lassen sich diese nicht holzschnittartig anhand von Hautpigmenten oder geografischer Herkunft charakterisieren.

\section{Nachweis der genetischen Vielfalt}

Der Nachweis der genetischen Vielfalt der Menschen hat Rassenkonzepte vollends ad absurdum geführt. „Beim Menschen besteht der mit Abstand größte Teil der genetischen Unterschiede nicht zwischen geographischen Populationen, sondern innerhalb solcher Gruppen“, so die Max-Planck-Gesellschaft für Menschheitsgeschichte. Vermeintliche menschliche Rassen gehen nachweislich nicht auf getrennte Evolutionslinien zurück. „Es gibt im menschlichen Genom unter den 3,2 Milliarden Basenpaaren keinen einzigen fixierten Unterschied, der etwa Afrikaner von Nicht-Afrikanern trennt. Es gibt - um es explizit zu sagen - somit nicht nur kein einziges Gen, welches, rassische Unterschiede begründet, sondern noch nicht mal ein einziges Basenpaar."

Dr. Thomas Meißner 
Hier steht eine Anzeige.

算 Springer 